\title{
The Laplacian Spread of a Tree
}

\section{Yi-Zheng Fan $^{1,2} \|$ Jing $\mathrm{Xu}^{1,2}$, Yi Wang ${ }^{1,2}$, Dong Liang $\|$}

${ }^{1}$ Key Laboratory of Intelligent Computing \& Signal Processing Ministry of Education of the People's Republic of China, Anhui University, Hefei 230039, P. R. China

非School of Mathematics and Computation Sciences, Anhui University, Hefei 230039, P. R. China

received September 20, 2007, revised March 1, 2008, accepted March 3, 2008.

The Laplacian spread of a graph is defined to be the difference between the largest eigenvalue and the second smallest eigenvalue of the Laplacian matrix of the graph. In this paper, we show that the star is the unique tree with maximal Laplacian spread among all trees of given order, and the path is the unique one with minimal Laplacian spread among all trees of given order.

Keywords: Tree, Laplacian matrix, Spread

\section{Introduction}

Let $G$ be a simple graph of order $n$ with the vertex set $V=V(G)=\left\{v_{1}, v_{2}, \ldots, v_{n}\right\}$ and the edge set $E=E(G)$. The adjacency matrix of the graph $G$ is defined to be a matrix $A=A(G)=\left[a_{i j}\right]$ of order $n$, where $a_{i j}=1$ if $v_{i}$ is adjacent to $v_{j}$, and $a_{i j}=0$ otherwise. Since $A$ is symmetric and real, the eigenvalues of $A$ can be arranged as follows:

$$
\lambda_{n}(G) \leq \lambda_{n-1}(G) \leq \cdots \leq \lambda_{1}(G) .
$$

The spread of the graph $G$ is defined as

$$
\mathscr{S}_{A}(G)=\lambda_{1}(G)-\lambda_{n}(G)
$$

Generally, the spread of a square complex matrix $M$ is defined to be $s(M)=\max _{i, j}\left|\lambda_{i}-\lambda_{j}\right|$, where the maximum is taken over all pairs of eigenvalues of $M$. There is a considerable literature on the spread of an arbitrary matrix, see, e.g. Johnson et al. (1985); Mirsky (1956); Nylen and Tam (1994); Thompson (1992); Zhan (2005).

\footnotetext{
${ }^{\dagger}$ Supported by National Natural Science Foundation of China (10601001), Anhui Provincial Natural Science Foundation (050460102, 070412065), Foundation of Innovation Team on Basic Mathematics of Anhui University.

${ }^{\ddagger}$ Supported by National Natural Science Foundation of China (60772121), NSF of Department of Education of Anhui Province (2005KJ005ZD), Foundation of Innovation Team on Eletrocircuit Design and Measure Technology of Anhui University.

$\S$ Current corresponding address.

1365-8050 @ 2008 Discrete Mathematics and Theoretical Computer Science (DMTCS), Nancy, France
} 
Recently, the spread of a graph has received much attention. In Petrović (1983), Petrović determines all minimal graphs whose spreads do not exceed 4. In Gregory et al. (2001), Gregory, Hershkowitz, and Kirkland present some lower and upper bounds for the spread of a graph. They show that the path is the unique graph with minimal spread among all connected graphs of given order. However the graph(s) with maximal spread is still unknown, and some conjectures are presented in their paper. In Li et al. (2007), $\mathrm{Li}$, Zhang and Zhou determine the unique graph with maximal spread among all unicyclic graphs with given order not less than 18 , which is obtained from a star by adding an edge between some two pendant vertices. In Nikiforov (2006), Nikiforov considers a more general problem: what is the property of the linear combination of some extreme eigenvalues of a graph? He gives a theorem involving the limit of a certain combination as the order of a graph goes to infinity, and presents an upper bound for the sum of the largest eigenvalue and the second largest eigenvalue of all graphs of fixed order.

In this paper, we consider another version of spread of a graph, i.e. the Laplacian spread of a graph, which is defined as follows. Let $G$ be a graph as above. The Laplacian matrix of a graph $G$ is defined as $L=L(G)=D(G)-A(G)$, where $D(G)=\operatorname{diag}\left\{d\left(v_{1}\right), d\left(v_{2}\right), \ldots, d\left(v_{n}\right)\right\}$ is a diagonal matrix, and $d(v)$ denotes the degree of a vertex $v$ of $G$. It is known that $L$ is symmetric and positive semidefinite so that its eigenvalues can be arranged as follows:

$$
0=\mu_{n}(G) \leq \mu_{n-1}(G) \leq \cdots \leq \mu_{1}(G),
$$

where $\mu_{n}(G)=0$ as each row sum of $L$ is zero. There are a lot of results involving with the relations between the spectrum of $L$ and numerous graph invariants, such as connectivity, diameter, isoperimetric number, and expansion properties of a graph; see, e.g., Fiedler (1973); Merris (1998); Mohar (1997). In particular, $\mu_{n-1}(G)>0$ if and only if $G$ is connected. Fiedler Fiedler (1973) calls the $\mu_{n-1}(G)$ the algebraic connectivity of the graph $G$, which is considered as an algebraic measurement of the connectivity of a graph. The corresponding eigenvector of $\mu_{n-1}(G)$ is usually called Fiedler vector, which has a beautiful structural property given by Fiedler (Fiedler. 1975, Theorem 3.14). One can find that $\mu_{1}(G)$ is exactly the spectral radius of $L$, which also has a lot of results (especially the upper bounds) for this eigenvalue; see e.g. Das (2003). The Laplacian spread of the graph $G$ is defined to be

$$
\mathscr{S}_{L}(G)=\mu_{1}(G)-\mu_{n-1}(G) .
$$

Note that in the definition we consider the largest eigenvalue and the second smallest eigenvalue, as the smallest eigenvalue always equals zero. In addition, throughout this paper we only consider connected graphs $G$ in which case $\mu_{n-1}(G)>0$.

We now consider the extremal Laplacian spread of trees. Let $P_{n}, S_{n}$ denote a path and a star of order $n$ respectively. It is known that for any tree $T$ of order $n$,

$$
\begin{gathered}
2\left(1+\cos \left(\frac{\pi}{n}\right)\right)=\mu_{1}\left(P_{n}\right) \leq \mu_{1}(T) \leq \mu_{1}\left(S_{n}\right)=n \text {, Chen (2002), } \\
2\left(1-\cos \left(\frac{\pi}{n}\right)\right)=\mu_{n-1}\left(P_{n}\right) \leq \mu_{n-1}(T) \leq \mu_{n-1}\left(S_{n}\right)=1 \text {, Fiedler (1973); Merris (1987); Chen (2002). }
\end{gathered}
$$

For both inequalities, the left equalities hold if and only if $T=P_{n}$, and the right equalities hold if and only if $T=S_{n}$. With equalities (1) and (2), we can not directly tell that $S_{n}$ is the one with maximal 


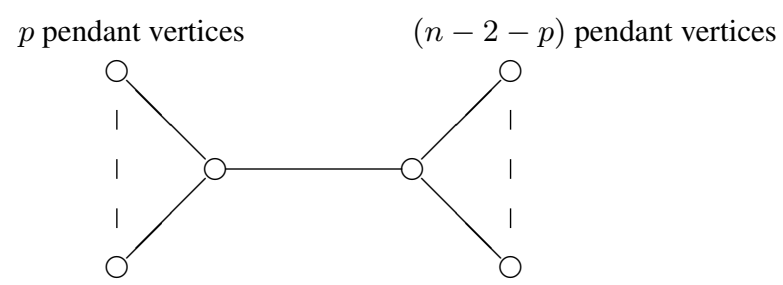

Fig. 1: A double star $\mathbf{T}(n, p)$ with $n-2 \geq p \geq(n-2) / 2$.

Laplacian spread among all trees of order $n$, and $P_{n}$ is the one with minimal Laplacian spread among all trees of order $n$.

We focus on the problem of maximizing and minimizing the Laplacian spread of trees, and show that $S_{n}$ and $P_{n}$ are indeed the trees respectively with maximal Laplacian spread and minimal Laplacian spread among all trees of order $n$. As the problem for a tree with order less than 5 is obvious, throughout the paper we assume all trees has order greater than or equal to 5 , unless specified somewhere.

\section{Tree(s) with maximal Laplacian spread}

We first narrow down the possibility of the tree(s) with maximal Laplacian spread.

Lemma 1 Anderson and Morel (1985) Let G be graph. Then

$$
\mu_{1}(G) \leq \max \{d(u)+d(v): u v \in E(G)\} .
$$

If $G$ is connected, then the equality holds if and only if $G$ is bipartite and the degree is constant on each partition of the vertices of $G$.

Denote by $\Delta(G)$ the maximum degree of all vertices of a graph $G$.

Lemma 2 Grone and Merris (1994) Let $G$ be graph of order $n \geq 2$ containing at least one edge. Then

$$
\mu_{1}(G) \geq \Delta(G)+1 .
$$

If $G$ is connected, then the equality holds if and only if $\Delta(G)=n-1$.

Let $T$ be the one with maximal Laplacian spread among all trees of order $n$. By Lemma 1 , we now prove $T$ is necessarily a double star of order $n$, i.e., a tree obtained from an edge by attaching $p$ pendant edges to one vertex of the edge and $n-2-p$ pendant edges to the other vertex of the edge with $n-2 \geq$ $p \geq(n-2) / 2$, denoted by $\mathbf{T}(n, p)$; see Fig. 1 .

Lemma 3 Let $T$ be the one with maximal Laplacian spread among all trees of order $n$. Then $T$ is a double star $\mathbf{T}(n, p)$ for some $p$ with $n-2 \geq p \geq(n-2) / 2$.

Proof: Let $T$ be a tree of order $n$, and let $N(v)$ denote the neighborhood of a vertex $v$ of $T$. Then for any edge $u v$ of $T$,

$$
d(u)+d(v)=|N(u) \cup N(v)| \leq n,
$$


with equality if and only if $T=\mathbf{T}(n, d(u)-1)$ (assuming $d(u) \geq d(v)$ ). In addition, the star $S_{n}=$ $\mathbf{T}(n, n-2)$ and $\mathscr{S}_{L}\left(S_{n}\right)=n-1$.

For any tree $T$ which is not a double star, then by above discussion and Lemma1 1 ,

$$
\mu_{1}(T) \leq \max \{d(u)+d(v): u v \in E(T)\} \leq n-1 .
$$

Then $\mathscr{S}_{L}(T)=\mu_{1}(T)-\mu_{n-1}(T)<n-1$ as $\mu_{n-1}(T)>0$. The result follows.

Proposition 1 The characteristic polynomial of $L(\mathbf{T}(n, p))$ is

$$
\operatorname{det}(\lambda I-L(\mathbf{T}(n, p)))=\lambda(\lambda-1)^{n-4}\left[(\lambda-n)(\lambda-1)^{2}+p(n-2-p) \lambda\right] .
$$

Proof: Let $q:=n-2-p$. By relabelling the vertices if necessary we may write the matrix $B:=$ $\lambda I-L(\mathbf{T}(n, p))$ as follows:

$$
B=\left[\begin{array}{cc|cc}
(\lambda-1) I_{p} & 0 & e_{p} & 0 \\
0 & (\lambda-1) I_{q} & 0 & e_{q} \\
\hline e_{p}^{T} & 0 & \lambda-(p+1) & 1 \\
0 & e_{q}^{T} & 1 & \lambda-(q+1)
\end{array}\right],
$$

where $I_{p}, e_{p}$ respectively denote an identity matrix of order $p$ and an all-one column vector of dimension $p$.

Assume that $\lambda \neq 1$. Then by left multiplying a block matrix $\left[\begin{array}{cc}I_{p+q} & 0 \\ -(\lambda-1)^{-1} \operatorname{diag}\left\{e_{p}^{T}, e_{q}^{T}\right\} & I_{2}\end{array}\right]$, the above matrix is transferred into

$$
\left[\begin{array}{cc|cc}
(\lambda-1) I_{p} & 0 & e_{p} & 0 \\
0 & (\lambda-1) I_{q} & 0 & e_{q} \\
\hline 0 & 0 & -p(\lambda-1)^{-1}+\lambda-(p+1) & 1 \\
0 & 0 & 1 & -q(\lambda-1)^{-1}+\lambda-(q+1)
\end{array}\right] .
$$

Hence

$$
\begin{aligned}
\operatorname{det} B & =(\lambda-1)^{p+q-2} \operatorname{det}\left[\begin{array}{cc}
-p+(\lambda-1)[\lambda-(p+1)] & (\lambda-1) \\
(\lambda-1) & -q+(\lambda-1)[\lambda-(q+1)]
\end{array}\right] \\
& =\lambda(\lambda-1)^{n-4}\left[(\lambda-n)(\lambda-1)^{2}+p(n-2-p) \lambda\right]
\end{aligned}
$$

Since the degree of the characteristic polynomial of $L(\mathbf{T}(n, p))$ is finite and there are infinite $\lambda(\lambda \neq 1)$ such that (3) holds, the result holds for all $\lambda \in \mathbb{R}$.

Denote

$$
f(\lambda ; n, p):=(\lambda-n)(\lambda-1)^{2}+p(n-2-p) \lambda .
$$

Then the characteristic polynomial of $L\left(S_{n}\right)$ of the star $S_{n}$ is $\lambda(\lambda-1)^{n-4} f(\lambda ; n, n-2)$. For any double star $\mathbf{T}(n, p)$ with $(n-2) / 2 \leq p \leq n-3$ and $n \geq 5$, by $(2),(0<) \mu_{n-1}(\mathbf{T}(n, p))<1$, and by Lemma 2. $\mu_{1}(\mathbf{T}(n, p)) \geq \Delta(\mathbf{T}(n, p))+1=p+2 \geq 4$. Hence the eigenvalues $\mu_{n-1}(\mathbf{T}(n, p)), \mu_{1}(\mathbf{T}(n, p))$ are 
both roots of the polynomial $f(\lambda ; n, p)$. In addition, all roots of $f(\lambda ; n, p)$ must be positive as they are nonzero eigenvalues of $L(\mathbf{T}(n, p))$.

In the following, we shall prove that for $(n-2) / 2 \leq p \leq n-3$ and $n \geq 5, \mathscr{S}_{L}(\mathbf{T}(n, p))<$ $\mathscr{S}_{L}(\mathbf{T}(n, n-2))=n-1$. By this inequality and Lemma 3 , we then get the result that the star is the unique tree with maximal Laplacian spread among all trees with given order. There are exactly two double stars $\mathbf{T}(5,2), \mathbf{T}(5,3)$ of order 5 , and by Lemma 4 above inequality holds for $n=5$. There are exactly three double stars of order 6 , i.e. $\mathbf{T}(6,2), \mathbf{T}(6,3), \mathbf{T}(6,4)$. By Lemma $4, \mathscr{S}_{L}(\mathbf{T}(6,3))<\mathscr{S}_{L}(\mathbf{T}(6,4))$. The inequality $\mathscr{S}_{L}(\mathbf{T}(6,2))<\mathscr{S}_{L}(\mathbf{T}(6,4))$ can be obtained directly by a little computation. By (4), $f(\lambda ; 6,2)=(\lambda-6)(\lambda-1)^{2}+4 \lambda$, which has roots $(5+\sqrt{17}) / 2,3,(5-\sqrt{17}) / 2$. So the Laplacian spread of $\mathbf{T}(6,2)$ is $\sqrt{17}<6-1=5$.

Lemma 4 For $n \geq 5$,

$$
\mathscr{S}_{L}(\mathbf{T}(n, n-3))<\mathscr{S}_{L}(\mathbf{T}(n, n-2))=\mathscr{S}_{L}\left(S_{n}\right)=n-1 .
$$

Proof: By Proposition 1 and the discussion prior to this lemma, the eigenvalues $\mu_{n-1}(\mathbf{T}(n, n-3)), \mu_{1}(\mathbf{T}(n, n-$ $3)$ ) are both roots of the polynomial

$$
f(\lambda ; n, n-3)=(\lambda-n)(\lambda-1)^{2}+(n-3) \lambda=\lambda^{3}-(n+2) \lambda^{2}+(3 n-2) \lambda-n .
$$

Note that

$$
f(1 / 3 ; n, n-3)=-\frac{23+3 n}{27}<0, \quad f(n-2 / 3 ; n, n-3)=\frac{4-39 n+9 n^{2}}{27}>0 \text { if } n \geq 5 .
$$

If there exists a $\lambda_{0}>n-2 / 3$ such that $f\left(\lambda_{0} ; n, n-3\right) \leq 0$ then $f(\lambda ; n, n-3)$ has two roots both greater than $n-2 / 3$, and hence the sum of its root is greater than $2 n-4 / 3$. However, the sum of all roots (necessarily be positive) of $f(\lambda ; n, n-3)$ is $n+2<2 n-4 / 3$, which is a contradiction, for $n \geq 4$. So $\lambda_{1}\left(\mathbf{T}(n, n-3)<n-2 / 3\right.$. Similarly, we get $\mu_{n-1}(\mathbf{T}(n, n-3)>1 / 3$. Hence

$$
\mathscr{S}_{L}(\mathbf{T}(n, n-3))<(n-2 / 3)-1 / 3=n-1 .
$$

The result follows.

Next we consider the Laplacian spread of $\mathbf{T}(n, p)$ with $(n-2) / 2 \leq p \leq n-4$.

Lemma 5 For $(n-2) / 2 \leq p \leq n-4$ and $n \geq 6$,

$$
\mathscr{S}_{L}(\mathbf{T}(n, p))<\mathscr{S}_{L}(\mathbf{T}(n, n-2))=\mathscr{S}_{L}\left(S_{n}\right)=n-1 .
$$

Proof: For brevity, we simply write $\mu_{1}(\mathbf{T}(n, p)), \mu_{n-1}(\mathbf{T}(n, p))$ as $\mu_{1}, \mu_{n-1}$ respectively. Observe that

$$
\mathscr{S}_{L}(\mathbf{T}(n, n-2))-\mathscr{S}_{L}(\mathbf{T}(n, p))=(n-1)-\left(\mu_{1}-\mu_{n-1}\right)=\left(n-\mu_{1}\right)-\left(1-\mu_{n-1}\right) .
$$

By 44, if $\lambda>0$, the image of $f(\lambda ; n, p)$ is obtained from $f(\lambda ; n, n-2)$ by adding a positive function $p(n-2-p) \lambda$; see Fig. 2 . 


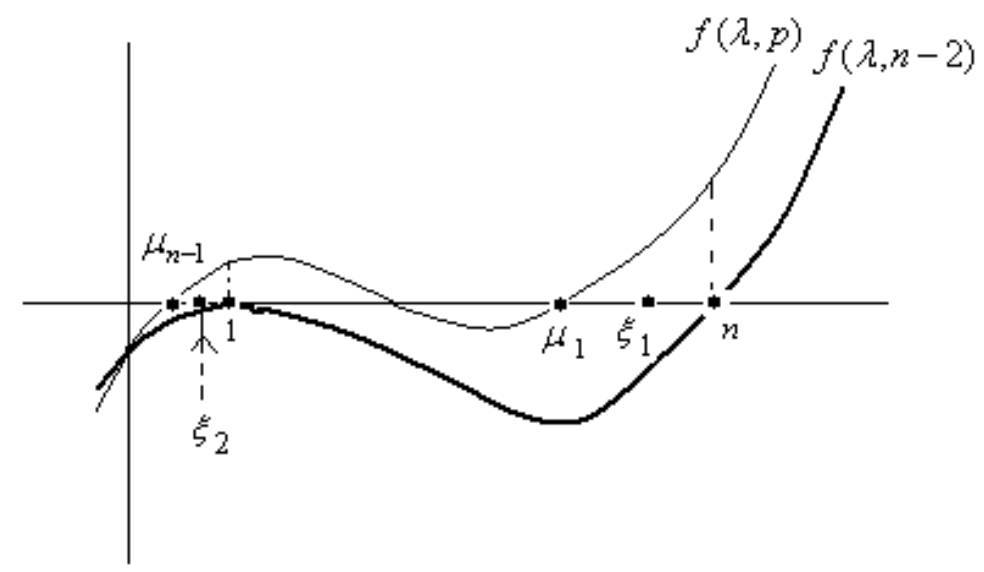

Fig. 2: The images of $f(\lambda ; n, p)$ with $(n-2) / 2 \leq p \leq n-3$ and $f(\lambda ; n, n-2)$.

By Mean-Value Theorem,

$$
\begin{gathered}
n-\mu_{1}=\frac{f(n ; n, p)-f\left(\mu_{1} ; n, p\right)}{f^{\prime}\left(\xi_{1} ; n, p\right)}=\frac{n p(n-2-p)}{f^{\prime}\left(\xi_{1} ; n, p\right)}, \\
1-\mu_{n-1}=\frac{f(1 ; n, p)-f\left(\mu_{n-1} ; n, p\right)}{f^{\prime}\left(\xi_{2} ; n, p\right)}=\frac{p(n-2-p)}{f^{\prime}\left(\xi_{2} ; n, p\right)},
\end{gathered}
$$

for some $\xi_{1} \in\left(\mu_{1}, n\right)$ and $\xi_{2} \in\left(\mu_{n-1}, 1\right)$, where $f^{\prime}(\lambda ; n, p)$ denotes the derivative of $f(\lambda ; n, p)$ with respect to $\lambda$. If we can show

$$
\frac{n p(n-2-p)}{f^{\prime}\left(\xi_{1} ; n, p\right)}>\frac{p(n-2-p)}{f^{\prime}\left(\xi_{2} ; n, p\right)}, \text { or } n f^{\prime}\left(\xi_{2} ; n, p\right)>f^{\prime}\left(\xi_{1} ; n, p\right)
$$

the result will follow.

Note that

$$
f^{\prime}(\lambda ; n, p)=3 \lambda^{2}-2(n+2) \lambda+2 n+1+p(n-2-p) .
$$

So $f^{\prime}(\lambda ; n, p)$ is strictly decreasing on the open interval $(0,(n+2) / 3)$ and is strictly increasing on $((n+$ $2) / 3),+\infty)$. Note that $0<\xi_{2}<1<(n+2) / 3$, and by Lemma $2, n>\xi_{1}>\mu_{1} \geq p+2 \geq$ $(n-2) / 2+2>(n+2) / 3$. Therefore,

$$
\begin{gathered}
n f^{\prime}\left(\xi_{2} ; n, p\right)>n f^{\prime}(1 ; n, p)=n p(n-2-p), \\
f^{\prime}\left(\xi_{1} ; n, p\right)<f^{\prime}(n ; n, p)=(n-1)^{2}+p(n-2-p) .
\end{gathered}
$$

Then

$$
n f^{\prime}\left(\xi_{2} ; n, p\right)-f^{\prime}\left(\xi_{1} ; n, p\right)>(n-1)[p(n-2-p)-(n-1)] .
$$

As $(n-2) / 2 \leq p \leq n-4, p(n-2-p)-(n-1) \geq 2(n-4)-(n-1)=n-7$. So, if $n \geq 7$, the result follows. If $n=6$, then $p=2$ and this case is verified prior to Lemma 4 .

By Lemmas 3,4 and 5 , we get the main result. 
Theorem 1 For $n \geq 5$, the star is the unique tree with maximal Laplacian spread among all trees of order $n$.

\section{Tree(s) with minimal Laplacian spread}

We first establish a relation between the Laplacian spread of a tree and the (adjacency) spread of the line graph of the tree, and then use the relation to obtain another main result of this paper.

Let $T$ be a tree of order $n$ with vertices $v_{1}, \ldots, v_{n}$ and edges $e_{1}, \ldots, e_{n-1}$. The (vertex-edge, unoriented) incidence matrix of $T$ is the $n \times(n-1)$ matrix $M(T)=\left[m_{i j}\right]$ given by: $m_{i j}$ equals 1 if vertex $v_{i}$ is incident to edge $e_{j}$ and equals 0 otherwise. The unoriented Laplacian matrix $K(T)$ of $T$ is defined to be the $n \times n$ matrix $K(T)=M(T) M(T)^{T}=D(T)+A(T)$.

The line graph of the tree $T$, denoted by $T^{l}$, is the graph whose vertices are exactly the edges of $T$ with two vertices being adjacent if and only if the the corresponding edges in $T$ are incident. One can find that $M(T)^{T} M(T)=2 I_{n-1}+A\left(T^{l}\right)$ (or see (Brualdi and Ryser. 1991, p. 35)). Note that $T^{l}$ is connected, and $P_{n}^{l}=P_{n-1}$ for $n \geq 3$.

Lemma 6 Grone et al. (1990) Let $G$ be a bipartite graph. Then $K(G)=D(G)+A(G)$ and $L(G)=$ $D(G)-A(G)$ are unitarily similar, i.e., there exists an orthogonal matrix $U$ such that $K(G)=U^{-1} L(G) U$.

Lemma 7 For any tree $T$ of order $n \geq 3$, the Laplacian spread of $T$ is exactly the (adjacency) spread of $T^{l}$, i.e.,

$$
\mathscr{S}_{L}(T)=\mathscr{S}_{A}\left(T^{l}\right) .
$$

Proof: As $T$ is bipartite, by Lemma 6 the matrix $K(T)=M(T) M(T)^{T}=D(T)+A(T)$ is unitarily similar to $L(T)=D(T)-A(T)$, and hence they have the same spectra. Note that $M(T) M(T)^{T}$ and $M(T)^{T} M(T)$ have the same set of nonzero eigenvalues, and $M(T)^{T} M(T)=2 I_{n-1}+A\left(T^{l}\right)$. Hence, the eigenvalues of $A\left(T^{l}\right)$ are $\mu_{1}(T)-2, \mu_{2}(T)-2, \ldots, \mu_{n-1}(T)-2$, and the Laplacian spread of $T$ is exactly the (adjacency) spread of $T^{l}$.

Lemma 8 Gregory et al. (2001) If $G$ is a connected graph of order $n$, then $\mathscr{S}_{A}(G) \geq \mathscr{S}_{A}\left(P_{n}\right)$, with equality if and only if $G=P_{n}$.

Theorem 2 For $n \geq 5$, the path is the unique tree with minimal Laplacian spread among all trees of $\operatorname{order} n$.

Proof: Let $T$ be any tree of order $n \geq 5$, which is not a path. Then $T^{l}$ is a connected graph of order $n-1$ which is not a path. Then by Lemmas 7 and 8 .

$$
\mathscr{S}_{L}(T)=\mathscr{S}_{A}\left(T^{l}\right)>\mathscr{S}_{A}\left(P_{n-1}\right)=\mathscr{S}_{A}\left(P_{n}^{l}\right)=\mathscr{S}_{L}\left(P_{n}\right) .
$$

The result follows.

\section{Acknowledgements}

The authors would like to thank the anonymous referees for valuable suggestions and corrections, which have improved the original manuscript. 


\section{References}

W. N. Anderson and T. D. Morel. Eigenvalues of the laplacian of a graph. Linear Multilinear Algebra, 18:141-145, 1985.

R. Brualdi and H. Ryser. Combinatorial Matrix Theory. Cambridge University Press, New York, 1991.

Y. Chen. Properties of spectra of graphs and line graphs. Appl. Math. J. Chinese Univ. Ser. B, 17(3):371-376, 2002.

K. Das. An improved upper bound for laplacian graph eigenvalues. Linear Algebra Appl., 368:269-278, 2003.

M. Fiedler. Algebraic connectivity of graphs. Czechoslovak Math. J., 23:298-305, 1973.

M. Fiedler. A property of eigenvectors of nonnegative symmetric matrices and its applications to graph theory. Czechoslovak Math. J., 25:619-633, 1975.

D. Gregory, D. Hershkowitz, and S. Kirkland. The spread of the spectrum of a graph. Linear Algebra Appl., 332-334: 23-35, 2001.

R. Grone and R. Merris. The laplacian spectrum of a graph II. SIAM J. Discrete Math., 7:229-237, 1994.

R. Grone, R. Merris, and V. Sunder. The laplacian spectrum of a graph. SIAM J. Matrix Anal. Appl., 11:218-238, 1990.

C. R. Johnson, R. Kumar, and H. Wolkowicz. Lower bounds for the spread of a matrix. Linear Algebra Appl., 71: 161-173, 1985.

X. Li, J. Zhang, and B. Zhou. The spread of unicyclic graphs with given size of maximum matchings. Journal of Mathematical Chemistry, 42:775-788, 2007.

R. Merris. Laplacian matrices of graphs: a survey. Linear Algebra Appl., 197/198:143-176, 1998.

R. Merris. Characteristic vertices of trees. Linear and Multilinear Algebra, 22:115-131, 1987.

L. Mirsky. The spread of a matrix. Mathematika, 3:127-130, 1956.

B. Mohar. Some applications of laplacian eigenvalues of graphs. In G. Hahn and G. Sabidussi, editors, Graph Symmetry, pages 225-275. Kluwer Academic Publishers, Dordrecht, 1997.

V. Nikiforov. Linear combinations of graph eigenvalues. Electronic Journal of Linear Algebra, 15:329-336, 2006.

P. Nylen and T. Y. Tam. On the spread of a hermitian matrix and a conjecture of thompson. Linear Multilinear Algebra, 37:3-11, 1994.

M. Petrović. On graph whose spectral spread does not exceed 4. Publications de l'institut mathématique, 34(48): 169-174, 1983.

R. C. Thompson. The eigenvalue spreads of a hermitian matrix and its principal submatrices. Linear Multilinear Algebra, 32:327-333, 1992.

X. Zhan. Extremal eigenvalues of real symmetric matrices with entries in an interval. SIAM. J. Matrix Anal. Appl., 27:851-860, 2005. 